Original research article

\title{
The effects of Rosmarinus officinalis L. essential oil and its nanoemulsion on dyslipidemic Wistar rats
}

\author{
Ana Paula Santos Rodrigues 1,2, Belmira Silva Faria e Souza ${ }^{1}$, Albenise Santana Alves Barros ${ }^{1,3}$, \\ Helison de Oliveira Carvalho ${ }^{1,3}$, Jonatas Lobato Duarte ${ }^{1,2}$, Letícia Elizandra Mehl Boettger ${ }^{2,6}$, \\ Robson Barbosa ${ }^{2,6}$, Adriana Maciel Ferreira ${ }^{1}$, Irlon Maciel Ferreira ${ }^{2,3,4}$, Caio Pinho Fernandes ${ }^{2,5}$, \\ Arlindo César Matias Pereira ${ }^{1}$, José Carlos Tavares Carvalho 1, 2, 3 * \\ ${ }^{1}$ Universidade Federal do Amapá, Departamento de Ciências Biológicas e da Saúde, Curso de Farmácia, Laboratório de Pesquisa em Fármacos, Macapá, \\ Amapá, Brasil \\ ${ }^{2}$ Universidade Federal do Amapá, Departamento de Ciências Biológicas e da Saúde, Programa de Pós-graduação em Ciências Farmacêuticas, Macapá, \\ Amapá, Brasil \\ ${ }^{3}$ Universidade Federal do Amapá, Departamento de Ciências Biológicas e da Saúde, Programa de Pós-graduação em Inovação Farmacêutica, Macapá, \\ Amapá, Brasil \\ ${ }^{4}$ Universidade Federal do Amapá, Curso de Química, Laboratório de Biocatálise e Biotransformação em Química Orgânica, Macapá, Amapá, Brasil \\ ${ }^{5}$ Universidade Federal do Amapá, Departamento de Ciências Biológicas e da Saúde, Curso de Farmácia, Laboratório de Nanobiotecnologia \\ Fitofarmacêutica, Macapá, Amapá, Brasil \\ ${ }^{6}$ Universidade Federal do Amapá, Departamento de Ciências Biológicas e da Saúde, Curso de Farmácia, Laboratório de Bioquímica e Citologia Clínica, \\ Macapá, Amapá, Brasil
}

\begin{abstract}
Dyslipidemias are lipid metabolism alterations that cause increased levels of serum lipoprotein, cholesterol, and triglycerides. These alterations are associated with a higher incidence of cardiovascular diseases and are a risk factor for atherosclerosis development. This study aimed to evaluate the effect of Rosmarinus officinalis essential oil (EORO, $100 \mathrm{mg} / \mathrm{kg}$ ) and its nanoemulsion (NEORO, $500 \mu \mathrm{gg} / \mathrm{kg}$ ) on Triton and coconut saturated-fat-induced (CSF) dyslipidemias using Wistar rats. The phytochemical evaluation of EORO performed by gas chromatography-mass spectroscopy (GC-MS) revealed 1,8-cineole (33.70\%), camphor (27.68\%), limonene (21.99\%), and $\alpha$-pinene (8.13\%) as its major compounds. Triton-induced dyslipidemia significantly increased total cholesterol, LDL, and triglycerides levels. On the other hand, the groups treated with EORO and NEORO had significantly reduced total cholesterol, LDL, and triglycerides compared to the group treated only with Triton. Similar results were observed on the positive control treated with simvastatin. Dyslipidemia induced with coconut saturated-fat (CSF) caused abdominal fat gain, hypercholesterolemia, hypertriglyceridemia, increased LDL levels, and atherogenesis in the aorta. In contrast, the groups treated with EORO, NEORO, and simvastatin had significantly reduced hypercholesterolemia and hypertriglyceridemia, reduced abdominal fat gain, and absence of atherogenesis in the vascular endothelium. Overall, in the Triton-induced dyslipidemia model, EORO treatment had superior values than NEORO's (and simvastatin), although the differences were not too high, while in the CSF model, the values were mixed. In this manner, our results show an anti-dyslipidemic and anti-atherogenic activity effect by EORO and NEORO.
\end{abstract}

Keywords: Anti-atherogenic; Anti-dyslipidemic; Essential oil; Nanoemulsion; Rosmarinus officinalis

\section{Highlights:}

- Treatments with EORO and NEORO were tested against Triton/CSF-induced dyslipidemia.

- The major compounds of the oil were 1,8-cineole, camphor, and limonene.

- The treatments improved biochemical parameters (TC, TG, HDL, LDL, AI)

- The treatments inhibited the formation of atheroma plaques in the aorta.

\footnotetext{
* Corresponding author: José Carlos Tavares Carvalho, Universidade Federal do Amapá, Departamento de Ciências Biológicas e da Saúde, Curso de Farmácia, Laboratório de Pesquisa em Fármacos, Rodovia Juscelino Kubitscheck, Km 02, CEP 68903-419, Macapá, Amapá, Brasil; e-mail: farmacos@unifap.br http://doi.org/10.32725/jab.2020.016

Submitted: 2019-03-21 • Accepted: 2020-10-18 • Prepublished online: 2020-11-06

J Appl Biomed 18/4: 126-135 • EISSN 1214-0287 • ISSN 1214-021X

(c) 2020 The Authors. Published by University of South Bohemia in České Budějovice, Faculty of Health and Social Sciences.

This is an open access article under the CC BY-NC-ND license.
} 


\section{Introduction}

Dyslipidemia consists of alterations on the lipids metabolism, resulting in increased low-density serum lipoproteins (LDL), cholesterol, triglycerides, and decreased high-density lipoproteins (HDL) - biomolecules that transport lipids in its core (Boullart et al., 2012; Brea Hernando, 2014; Dowla et al., 2018). The pathology is linked to an increased incidence of cardiovascular diseases (CVD), diabetes, metabolic syndrome, and it is a risk factor for atherosclerosis (Dong et al., 2018; Lin et al., 2018). CVDs represent a central public health issue, and every year there is an increase of 12 million people affected; the difficulty of treatment is a risk factor for early death (Souza et al., 2017a).

Atherosclerosis, in turn, is a progressive inflammatory disease that affects medium to large-caliber arteries. The condition is triggered by an endothelial injury, followed by lipid deposition, muscle-cells migration, and calcification, mainly in the arteries' tunica intima (Bonfim et al., 2015).

In this context, biodiversity stands out as a great source of plant species and bioactive molecules with therapeutic potential for several diseases, including dyslipidemias. The plant species Rosmarinus officinalis L., popularly known as "Rosemary", belongs to the Lamiaceae family (formerly Labiatae). Original of southern Europe, North Africa, and the Mediterranean, $R$. officinalis is nowadays cultivated worldwide and presents several pharmacological activities such as anti-inflammatory, anti-bacterial, and antioxidant (Satyal et al., 2017; Sedighi et al., 2015). The essential oil of R. officinalis (EORO) is a colorless or pale-yellow liquid with the plant's characteristic scent (Rašković et al., 2014). Its chemical composition can differ according to the soil, farming factors, and extraction method (Borges et al., 2017). According to Cleff et al. (2012) and Takayama et al. (2016), the major compounds of this oil include 1,8-cineol, $\alpha$-pinene, and limonene. Due to its anti-bacterial and antioxidant potential, EORO is widely used in the pharmaceutical, cosmetics, and food industries (Affholder et al., 2013).

A novel approach of using essential oils is in the form of nanoemulsions. These formulations can boost the pharmacological actions from essential oils due to the nanometric scale of the droplets. The development of nanoemulsions from immiscible liquids can be achieved by adding a stabilizer, which enables kinetic stability by forming small droplets whose sizes range from 20 to $200 \mathrm{~nm}$ (Ostertag et al., 2012). In this study, we aimed to evaluate the effect of Rosmarinus officinalis L. essential oil and its nanoemulsion over dyslipidemia. For this, we used the model of dyslipidemia induced by coconut saturated fat and Triton in Wistar rats.

\section{Materials and methods}

\section{Essential oil of Rosmarinus officinalis L. (EORO)}

The essential oil was acquired from the company Florien Flowers and Herbs Pharmaceutical Commerce Ltda. Located at Vicente Bellini road, 175, Piracicaba, São Paulo, Brazil. The oil was extracted from plants' aerial parts, with botanical identification lot no 056757.

\section{Phytochemical characterization of EORO by gas chromatography-mass spectroscopy (GC-MS)}

The chemical composition of EORO was analyzed through gas chromatography-mass spectroscopy using a Shimadzu equip- ment (GC 2010), with a self-injector (Shimadzu AOC-5000), a mass-detector (Shimadzu MS2010 Plus, $70 \mathrm{eV}$ ), and a DB$5 \mathrm{MS}$ fused silica column (Agilent Advanced J \& W; $30 \mathrm{~m} \times$ $0,25 \mathrm{~mm} \times 0,25 \mu \mathrm{m})$. The split-ratio was set at 1:30; helium was used as the carrier gas $(65 \mathrm{kPa})$; the injection volume was $1.0 \mu \mathrm{l}$, and the injector temperature was set at $250{ }^{\circ} \mathrm{C}$. The detector temperature also was set at $250{ }^{\circ} \mathrm{C}$; the initial column temperature was set at $60{ }^{\circ} \mathrm{C}$ for $1 \mathrm{~min}$, heating $3{ }^{\circ} \mathrm{C} \mathrm{min}-1$ until $290^{\circ} \mathrm{C}$.

The analysis lasted $46.67 \mathrm{~min}$, and the compounds were identified through their retention index (RI), interpolating their retention time based on a mixture of aliphatic hydrocarbons (C9-C30) analyzed at the same conditions. Also, the compounds' fragmentation pattern was compared to the equipment's mass spectrum library (NIST 5.0).

\section{Cocos nucifera saturated fat (CSF)}

The coconut oil was purchased from Cocos Empire Company Municipal Market of Belo Horizonte, Minas Gerais, Brazil. The oil extraction was performed through endocarp pressing, followed by water addition in $1: 1(\mathrm{v} / \mathrm{v})$ proportion. We separated the fat from the oil by heating at $80^{\circ} \mathrm{C}$, as described by Handayani et al. (2009).

\section{EORO nanoemulsion preparation}

The EORO nanoemulsion (NEORO) was prepared using a low energy-load method, described previously by Fernandes et al. (2013). For a final mass of $50 \mathrm{~g}$, we used water (90\%), EORO (5\%), and Tween-20 (5\%) as the surfactant.

One phase was initially prepared by mixing EORO and Tween-20; this mixture was stirred using a magnetic stirrer (Even HJ-4) over 30 minutes at $750 \mathrm{rpm}$. Then, the aqueous phase was added at $0.5 \mathrm{ml} / \mathrm{min}$ flow under continuous stirring over 60 minutes. The nanoemulsion was assessed 0,1 , and 7 days after its preparation for the following parameters: macroscopic color and visual aspect, phases separation, and sedimentation. Moreover, the droplets size analysis and polydispersity index were evaluated using a Zetasizer (Malvern, Nano Series) according to described by Borges et al. (2017). The NEORO was kept at room temperature $\left(25 \pm 2{ }^{\circ} \mathrm{C}\right)$ in capped test tubes.

\section{Animals and ethical aspects}

The Ethics Committee in Animals Use (CEUA) from the Federal University of Amapá approved this study under no 017/2017.

The animals used were male Wistar rats (Rattus norvegicus albinus) from the Animal Investigation Multidisciplinary Center (CEMIB) of Campinas University - UNICAMP. The rats were maintained in polyethylene cages placed in a ventilated cabinet at controlled temperature $\left(25 \pm 2{ }^{\circ} \mathrm{C}\right)$, dark/light cycle (12/12 hours), and received food and water in controlled quantity.

\section{Triton-induced dyslipidemia model}

In this experiment, the animals were randomly assigned into five groups ( $n=6$ per group) and orally treated for 5 days, according to their groups. The dyslipidemia was induced on the second day by injecting Triton intraperitoneally (WR1339, Tyloxapol, Sigma-Aldrich) at $150 \mathrm{mg} / \mathrm{kg}$ dissolved in saline solution (Souza et al., 2017a). EORO and NEORO doses were chosen based on Borges et al. (2017). The groups were designed as follows:

- Control Group: Treated with $0.5 \mathrm{ml}$ of Tween-20 (4\%) solution; did not receive Triton treatment; 
- Triton+VEI Group: Treated with $0.5 \mathrm{ml}$ of Tween-20 (4\%) solution (vehicle) and Triton;

- Triton+EORO Group: Treated with $100 \mathrm{mg} / \mathrm{kg}$ of EORO with $0.5 \mathrm{ml}$ of Tween-20 (4\%) as a vehicle and Triton;

- Triton+NEORO Group: Treated with $500 \mu \mathrm{g} / \mathrm{kg}$ of NEORO and Triton;

- Triton+SIM Group: Treated with Simvastatin 10 mg/kg and simvastatin.

\section{CSF-induced dyslipidemia model}

Animals were randomly assigned into five groups ( $n=7$ per group) and orally treated according to the CSF dyslipidemia-inducing method described by Souza et al. (2017b). EORO and NEORO doses were chosen based on Borges et al. (2017). The groups were designed as follows:

- CSF+VEI Group: Treated with $0.5 \mathrm{ml}$ of Tween-20 (4\%) solution (vehicle) for 40 days, and $2 \mathrm{ml}$ of CSF from the 20th to the 40th day;

- EORO Group: Treated with $100 \mathrm{mg} / \mathrm{kg} /$ day of EORO for 40 days;

- CSF+EORO Group: Treated with $100 \mathrm{mg} / \mathrm{kg}$ of EORO for 40 days, and $2 \mathrm{ml}$ of CSF from the 20th to the 40th day;

- CSF+NEORO Group: Treated with $500 \mu \mathrm{g} / \mathrm{kg}$ of NEORO for 40 days, and $2 \mathrm{ml}$ of CSF from the 20th to the 40th day;

- CSF+SIM Group: Treated with Simvastatin $20 \mathrm{mg} / \mathrm{kg}$ for 40 days, and $2 \mathrm{ml}$ of CSF from the 20th to the 40th day.

\section{Biochemical analysis}

In the CSF-induced dyslipidemia model, the animals were kept fasting for 12 hours on the 41st day to collect blood samples. For this, they were anesthetized using $45 \mathrm{mg} / \mathrm{kg}$ of sodium thiopental (Cristália - Chemical and Pharmaceutical Products Ltda, Brazil) intraperitoneally.

Blood samples $(1.5 \mathrm{ml})$ were collected from the ocular plexus and centrifuged over 10 minutes (5000 rpm) for analysis of aspartate aminotransferase (AST), alanine aminotransferase (ALT), total cholesterol (TC) and fractions (LDL and HDL), tri- glycerides (TG), urea, glucose, and creatinine. The Atherogenic (AI) index was calculated as described by Dobiášová (2004):

$$
\text { Log(Triglycerides/HDL-Cholesterol) }
$$

In the Triton-induced dyslipidemia model, the animals were treated as previously described, but on the fifth day. All tests were performed using LabTest kits and automated biochemical analyzer equipment model BS 380 (Mindray Bio-medical Electronics Co., Ltd.).

\section{Organs removal and aorta scanning electron microscopy (SEM)}

On the 41st day of the CSF-induced dyslipidemia, the animals were euthanized by Thiopental overdose (Cristália Ltda, Brazil) to remove their organs (kidney, spleen, liver, pancreas, lungs, and abdominal fat), which were weighed using an electronic analytical balance (Model Bioprecisa FA-2104N). The aorta was removed from the aortic arch to the iliac bifurcation. The thoracic region was divided into $0.5 \mathrm{~cm}$ sections for analysis using an SEM equipment (Hitachi Model-TM3030 PLUS) to search for atherogenic processes, as described by Souza et al. (2017b).

\section{Statistical analysis}

The results from the experiments were expressed as a mean \pm standard deviation. The groups were compared using Analysis of Variance (ANOVA One-Way) followed by the post-hoc Tukey test, and $p<0.05$ was considered statistically significant.

\section{Results}

\section{EORO's chemical profile (GC-MS)}

The chromatography showed $100 \%$ of terpenes in EORO composition, with 20 compounds identified. The major compounds were $\alpha$-pinene (8.13\%), limonene (21.99\%), 1,8-cineole (33.70), and camphor (27.68\%), as shown in Fig. 1 and Table 1.

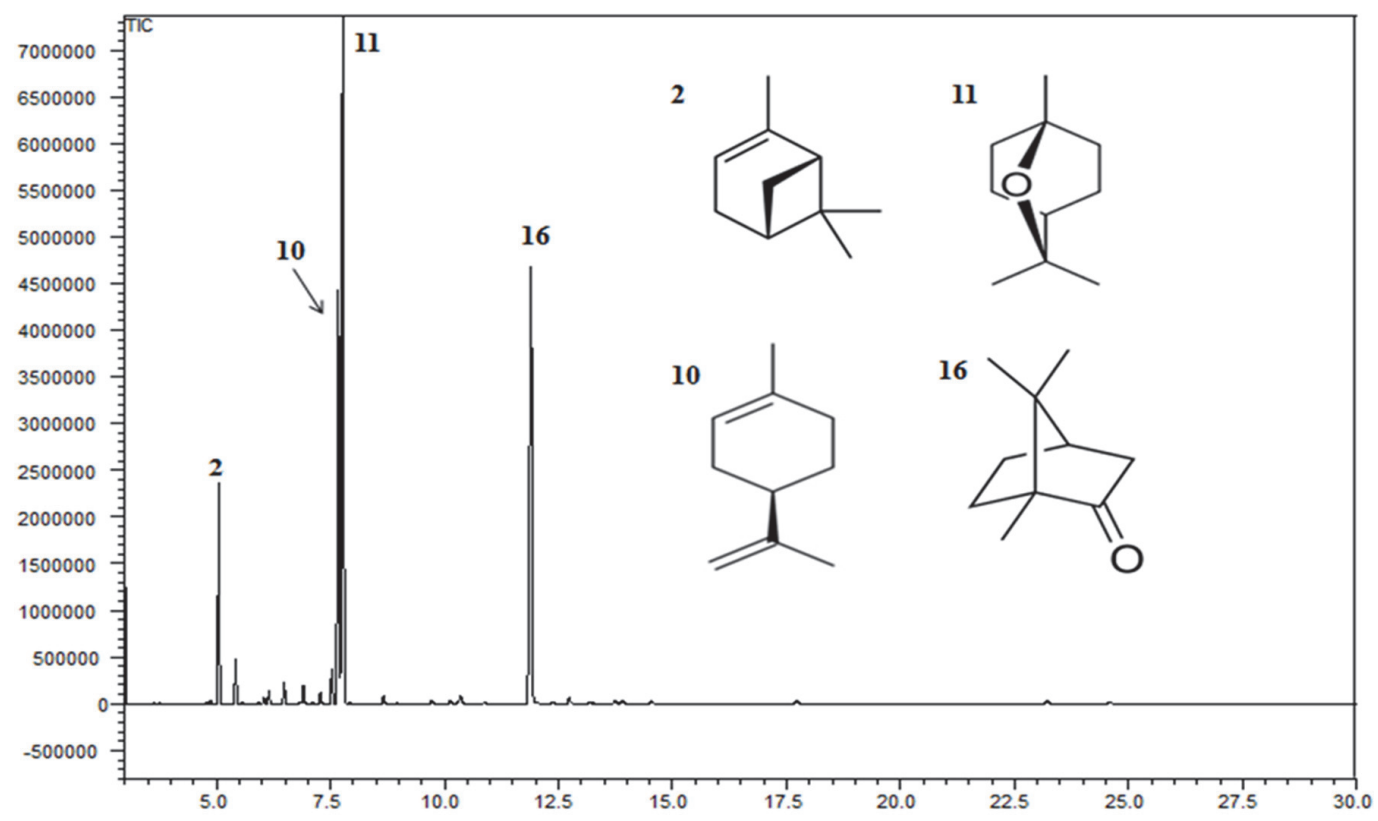

Fig. 1. Chromatographic profile (GC-MS) from the essential oil of Rosmarinus officinalis (EORO). (2) $\alpha$-pinene (8.13\%); (10) Limonene (21.99\%); (11) 1,8-cineole (33.70\%); (16) camphor (27.68\%). 
Table 1. Chemical constituents identified in EORO through GC-MS

\begin{tabular}{|c|c|c|c|c|}
\hline Peak & $\mathrm{RT}(\min )$ & Compound & $(\%)$ & RI \\
\hline 1 & 4.872 & $\alpha$-thujene & 0.11 & 928 \\
\hline 2 & 5.054 & $\alpha$-pinene & 8.13 & 935 \\
\hline 3 & 5.424 & Camphene & 1.68 & 950 \\
\hline 4 & 6.045 & $\beta$-phellandrene & 0.21 & 955 \\
\hline 5 & 6.152 & $\beta$-pinene & 0.58 & 979 \\
\hline 6 & 6.482 & $\beta$-myrcene & 0.90 & 993 \\
\hline 7 & 6.911 & $\alpha$-phellandrene & 0.77 & 1007 \\
\hline 8 & 7.282 & $\alpha$-terpinene & 0.45 & 1018 \\
\hline 9 & 7.532 & o-cymene & 1.65 & 1026 \\
\hline 10 & 7.674 & Limonene & 21.99 & 1030 \\
\hline 11 & 7.773 & 1,8-cineole & 33.70 & 1033 \\
\hline 12 & 8.666 & $\gamma$-terpinene & 0.39 & 1059 \\
\hline 13 & 9.724 & Terpinolene & 0.20 & 1091 \\
\hline 14 & 10.128 & $\beta$-linalool & 0.16 & 1102 \\
\hline 15 & 10.350 & $* * *$ & 0.44 & 1108 \\
\hline 16 & 11.897 & Camphor & 27.68 & 1147 \\
\hline 17 & 12.736 & Borneol & 0.32 & 1168 \\
\hline 18 & 13.739 & $\alpha$-terpineol & 0.12 & 1193 \\
\hline 19 & 13.899 & $\alpha$-campholenal & 0.20 & 1197 \\
\hline 20 & 14.532 & Verbenone & 0.18 & 1213 \\
\hline \multirow[t]{2}{*}{21} & 23.220 & $\beta$-caryophyllene & 0.14 & 1421 \\
\hline & & Identification percentage & 100.00 & \\
\hline
\end{tabular}

RT - Retention time; RI - Index retention.

\section{NEORO assessment}

The nanoemulsion from EORO (NEORO) had a white coloration with slightly bluish reflect, no phase separation was observed nor any other parameter indicating instability, as described by Duarte et al. (2015). As for NEORO sta- bility evaluation over 7 days (Table 2 and Fig. 2), it was observed that average droplet size ranged from $129.1 \pm 0.35$ to $149.7 \pm 0.3786 \mathrm{~nm}$, and the polydispersity index ranged between $0.103 \pm 0.023$ and $0.376 \pm 0.005$.

Table 2. Particle size and polydispersity index of the NEORO triplicates evaluated in days 0,1 , and 7 . The results represent the mean \pm standard deviation (SD)

\begin{tabular}{|c|c|c|c|}
\hline & Day 0 & Day 1 & Day 7 \\
\hline \multirow{3}{*}{ Size (nm) } & 129.1 & 149.4 & 146.7 \\
\hline & 129.5 & 149.5 & 147.9 \\
\hline & 128.8 & 150.1 & 152.2 \\
\hline Mean \pm SD & $129.1 \pm 0.35$ & $149.7 \pm 0.3786$ & $148.9 \pm 2.892$ \\
\hline \multirow{3}{*}{ Polydispersity index } & 0,096 & 0,207 & 0,376 \\
\hline & 0,085 & 0,187 & 0,381 \\
\hline & 0,129 & 0,188 & 0,371 \\
\hline Mean \pm SD & $0.103 \pm 0.023$ & $0,194 \pm 0.011$ & $0,376 \pm 0,005$ \\
\hline
\end{tabular}

\section{Effect of EORO and NEORO treatment on the Triton- induced dyslipidemia model}

The group treated only with Triton had significantly increased TC $(192.8 \pm 29.64 \mathrm{mg} / \mathrm{dl})$ and TG $(245.7 \pm 26.6 \mathrm{mg} / \mathrm{dl})$ compared to the control group (Fig. 3). However, compared to the Triton-treated group, the Triton+EORO group had a significant reduction of serum TC $(68.3 \%, 109.66 \pm 45.25 \mathrm{mg} / \mathrm{dl})$ and TG $(94.8 \%, 97.33 \pm 25.82 \mathrm{mg} / \mathrm{dl})$. This was also observed in the Triton+NEORO group (TC reduction: $55.3 \%, 125.25 \pm$ $43.81 \mathrm{mg} / \mathrm{dl}$; TG reduction: $66.8 \%, 142.22 \pm 45.93 \mathrm{mg} / \mathrm{dl})$. Besides, the simvastatin-treated group (Triton+SIM) also had a reduction of serum TC and TG levels, as expected $(65.9 \%$, $112.25 \pm 44.19 \mathrm{mg} / \mathrm{dl}$, and 67.3\%, $141.3 \pm 22.10 \mathrm{mg} / \mathrm{dl}$, respectively). 

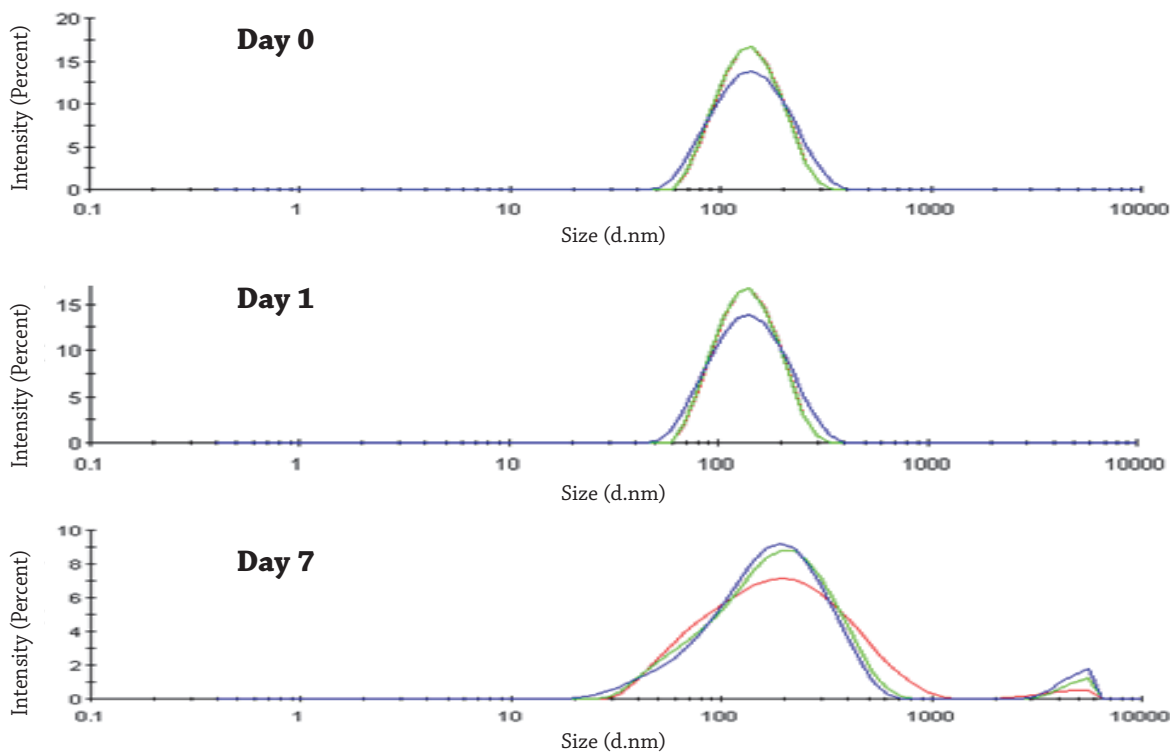

Fig. 2. Distribution of NEORO nanoparticle sizes assessed through a Zetasizer in days 0,1 , and 7.
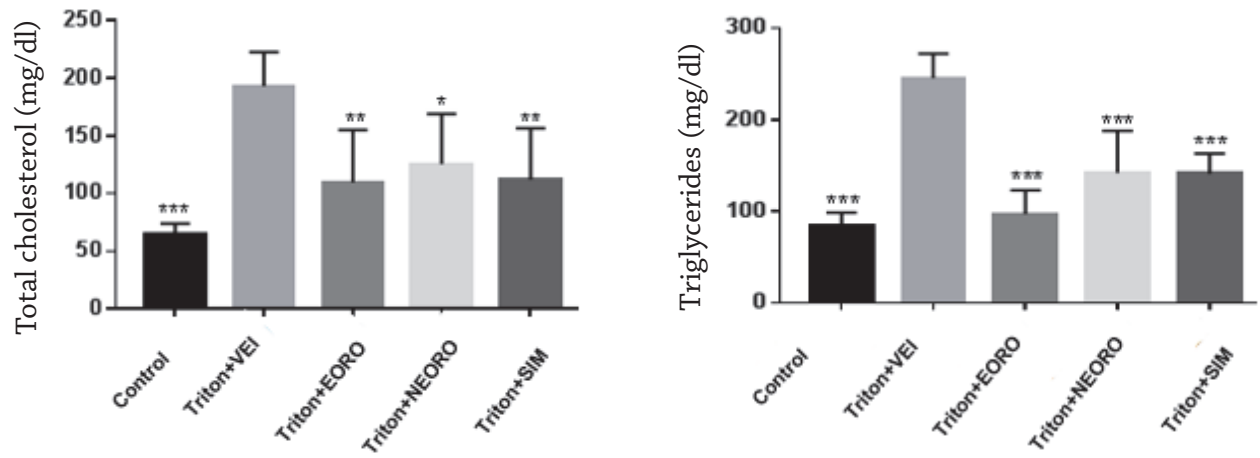

Fig. 3. Effect of the treatments on total. Cholesterol and triglycerides levels on the Triton-induced dyslipidemia model. Bars represent the mean \pm SD ( $n=7 /$ group). ${ }^{*} p<0.05 ;{ }^{* *} p<0.01 ;{ }^{* * *} p<0.001$, compared to the Triton+VEI group, One-Way ANOVA, with Tukey's as post-hoc test.

Triton induced significant LDL and HDL changes (95.2 \pm $13.6 \mathrm{mg} / \mathrm{dl}$ and $13.8 \pm 5.6 \mathrm{mg} / \mathrm{dl}$, respectively), as shown in Fig. 4. On the other hand, the groups who received EORO and NEORO had significantly reduced LDL $(67.7 \%, 51.6 \pm 18.3$ $\mathrm{mg} / \mathrm{dl}$ and $53.9 \%, 60.7 \pm 21.03 \mathrm{mg} / \mathrm{dl}$, respectively) and in-

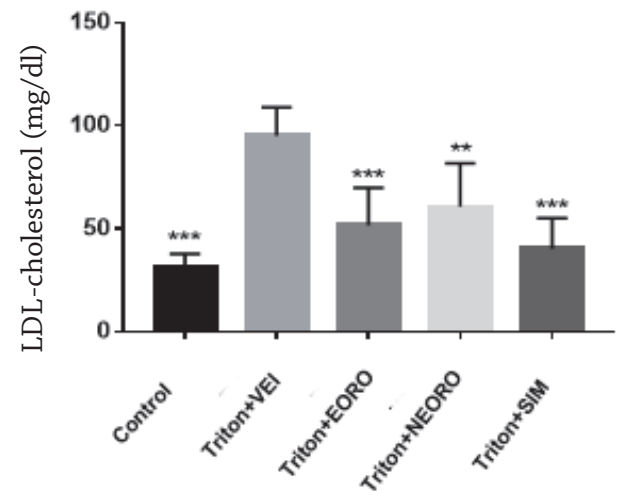

creased HDL levels (109.5\%, $44.66 \pm 16.2 \mathrm{mg} / \mathrm{dl}$ and $90.4 \%$, $40.2 \pm 13.7 \mathrm{mg} / \mathrm{dl}$, respectively). The group treated with the control drug simvastatin also had significantly reduced LDL $(84.2 \%, 40.314 .9 \mathrm{mg} / \mathrm{dl})$ and increased HDL levels $(76.2 \%$, $37.2 \pm 5.33 \mathrm{mg} / \mathrm{dl})$.

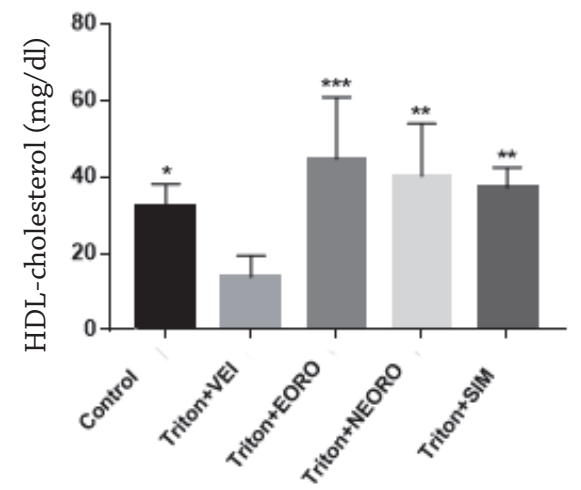

Fig. 4. Effect of treatments with EORO and NEORO on LDL and HDL in Triton-induced dyslipidemia. Bars represent the mean \pm SD $(n=$ 7/group). One-way ANOVA test followed by the post-hoc Tukey's test. ${ }^{*} p<0.05$; ${ }^{* *} p<0.01 ;{ }^{* * *} p<0.001$ compared to the Triton+VEI group. 


\section{Effect of EORO and NEORO treatment on the CSF-} induced dyslipidemia model

In CSF-induced dyslipidemia, bodyweight evaluation showed no statistically significant difference between groups (Fig. 5); however, abdominal fat appraisal shows that group CSF+VEI had higher values than the other groups. Treated groups $\mathrm{CSF}+\mathrm{EORO}, \mathrm{CSF}+\mathrm{NEORO}$, and $\mathrm{CSF}+\mathrm{SIM}$ had significantly reduced fat accumulation compared to group CSF+VEI. The internal organs weigh (Table 3 ) had no statistical difference among groups.
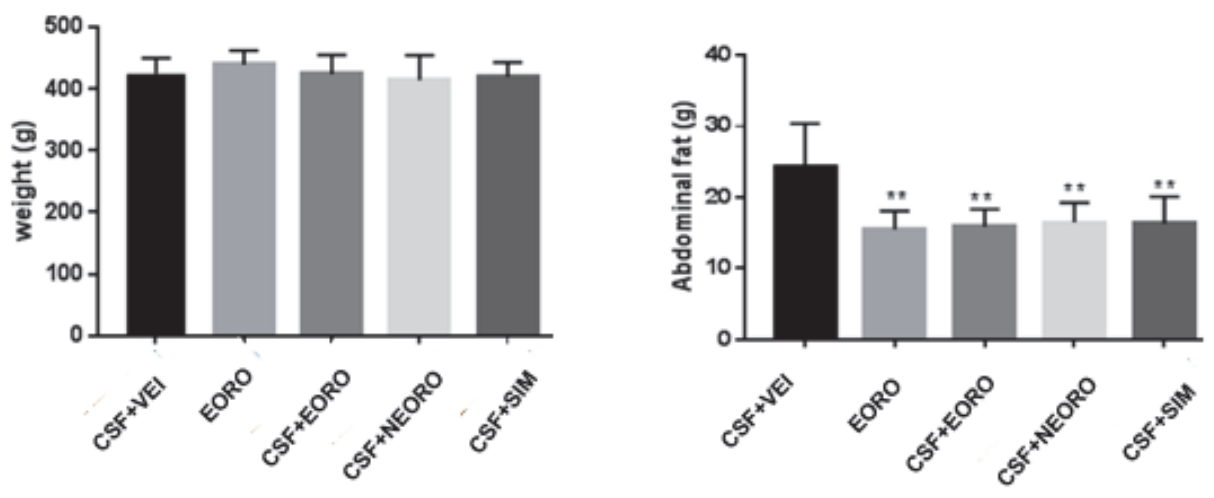

Fig. 5. Effect EORO and NEORO treatments on body weight and abdominal fat of Wistar rats with CSF-induced dyslipidemia. Bars represent the mean $\pm \mathrm{SD}$ ( $n=7$ /group). One-way ANOVA test followed by the post-hoc Tukey's test. ${ }^{*} p<0.05 ;{ }^{* *} p<0.01 ;{ }^{* * *} p<0.001$ compared to the CSF+VEI group.

Table 3. Effect of EORO and NEORO treatments on Wistar rats' organs weight with CSF-induced dyslipidemia

\begin{tabular}{|c|c|c|c|c|c|}
\hline Organs & $\mathrm{CSF}+\mathrm{VEI}$ & EORO & $\mathrm{CSF}+\mathrm{EORO}$ & $\mathrm{CSF}+\mathrm{NEORO}$ & $\mathrm{CSF}+\mathrm{SIM}$ \\
\hline Liver & $17.14 \pm 1.11$ & $16.41 \pm 0.28$ & $15.90 \pm 1.61$ & $16.77 \pm 2.38$ & $16.29 \pm 1.58$ \\
\hline Kidneys & $1.63 \pm 0.10$ & $1.83 \pm 0.17$ & $1.62 \pm 0.14$ & $1.83 \pm 0.19$ & $1.64 \pm 0.14$ \\
\hline Pancreas & $1.15 \pm 0.32$ & $1.37 \pm 0.33$ & $1.22 \pm 0.17$ & $1.48 \pm 0.19$ & $1.50 \pm 0.31$ \\
\hline Heart & $1.37 \pm 0.12$ & $1.54 \pm 0.20$ & $1.42 \pm 0.11$ & $1.49 \pm 0.15$ & $1.32 \pm 0.07$ \\
\hline Spleen & $0.99 \pm 0.05$ & $1.11 \pm 0.15$ & $0.94 \pm 0.09$ & $1.11 \pm 0.06$ & $1.08 \pm 0.11$ \\
\hline Lungs & $1.98 \pm 0.17$ & $2.04 \pm 0.16$ & $1.93 \pm 0.16$ & $2.12 \pm 0.12$ & $1.94 \pm 0.21$ \\
\hline
\end{tabular}

No statistical differences were observed (One-way ANOVA).

The biochemical parameters (Table 4) of CSF-induced dyslipidemic animals had no significant differences in transaminases levels (AST and ALT) among groups. However, as for TC was observed increased levels in the CSF+VEI group $(116.57 \pm 7.69 \mathrm{mg} / \mathrm{dl})$, while a highly significant reduction was observed in treated groups CSF+EORO, CSF+NEORO, and CSF+SIM (36.1\%, $42.5 \%$, and $50.1 \%$ reduction, respectively). The HDL values had no statistical differences among groups, evidencing no influence of the treatments on this parameter. However, LDL values were increased in the group CSF+VEI $(44.71 \pm 11.14 \mathrm{mg} / \mathrm{dl})$, with a significant reduction in CSF+ EORO and CSF+NEORO groups when compared to CSF+VEI $(64.3 \%, 15.71 \pm 8.88 \mathrm{mg} / \mathrm{dl}$, and 61.7\%, $16.85 \pm 8.06 \mathrm{mg} /$ $\mathrm{dl}$, respectively). The simvastatin-treated group (CSF+SIM) also had significant reduction of LDL levels (83.9\%, $7.10 \pm$ $3.11 \mathrm{mg} / \mathrm{dl})$.

In the CSF+VEI group was observed increased values of serum TG $(226.82 \pm 36.94 \mathrm{mg} / \mathrm{dl})$, while the treated groups $\mathrm{CSF}+\mathrm{EORO}$ and $\mathrm{CSF}+\mathrm{NEORO}$ had significantly reduced TG levels compared to it $(38.4 \%, 139.28 \pm 29.73 \mathrm{mg} / \mathrm{dl}$ and $41.9 \%, 131.28 \pm 40.58 \mathrm{mg} / \mathrm{dl}$, respectively). The simvastatin-treated group (CSF+SIM), although significantly reducing TG levels compared to CSF+VEI, had a lower reduction (31.9\%) compared to the former groups. No significant difference was observed for creatinine, glucose, and urea levels among groups. As for the Atherogenic Index (AI), it was observed that the treated groups $\mathrm{CSF}+\mathrm{EORO}, \mathrm{CSF}+\mathrm{NEORO}$, and $\mathrm{CSF}+\mathrm{SIM}$ all were statistically different from CSF+VEI (Table 4).

Scanning electron microscopy of the aorta shows atherogenic processes in the vascular endothelium found in the group CSF+VEI (Fig. 6 and 7). While on treated groups, CSF+EORO, $\mathrm{CSF}+\mathrm{NEORO}$, and CSF+SIM, atherogenesis was not observed.

\section{Discussion}

EORO composition is based on volatile compounds, mainly monoterpenes (Fernandes et al., 2013). Its characterization can be assessed through chromatographic and spectroscopic techniques to identify and quantify its major compounds (Carvalho et al., 2016). Our essential oil from R. officinalis was composed mainly of 1,8-cineole (33.70\%), camphor (27.68\%), limonene (21.99\%), and $\alpha$-pinene (8.13\%), with is similar with the literature reporting $\alpha$-pinene, camphor, 1,8 -cineole, and limonene as major compounds (Fernandes et al., 2013; Borges et al., 2017). 
Table 4. Effect of treatments with EORO and NEORO on biochemical parameters of Wistar rats with CSF-induced dyslipidemia

\begin{tabular}{lccccc}
\hline Parameters & CSF+VEI & EORO & CSF+EORO & CSF+NEORO & CSF+SIM \\
\hline AST (U/dl) & $87.28 \pm 12.97$ & $85.08 \pm 11.93$ & $70.0 \pm 9.52$ & $90.28 \pm 23.76$ & $78.71 \pm 9.08$ \\
ALT (U/dl) & $43.28 \pm 8.47$ & $28.55 \pm 4.61$ & $33.3 \pm 13.06$ & $35.71 \pm 6.79$ & $34.2 \pm 3.91$ \\
TC (mg/dl) & $116.57 \pm 7.69$ & $59.1 \pm 8.20^{\#}$ & $74.28 \pm 9.72^{\#}$ & $66.71 \pm 15.61^{\#}$ & $58.14 \pm 6.79^{\#}$ \\
HDL (mg/dl) & $31.0 \pm 10.66$ & $34.16 \pm 9.84$ & $31.14 \pm 6.56$ & $26.28 \pm 10.15$ & $32.5 \pm 5.32$ \\
LDL (mg/dl) & $44.71 \pm 11.14$ & $9.5 \pm 6.18^{\#}$ & $15.71 \pm 8.88^{\#}$ & $16.85 \pm 8.06^{\#}$ & $7.10 \pm 3.11^{\#}$ \\
Creatinine (mg/dl) & $0.22 \pm 0.04$ & $0.27 \pm 0.03$ & $0.24 \pm 0.03$ & $0.20 \pm 0.02$ & $0.20 \pm 0.01$ \\
Glucose (mg/dl) & $134.85 \pm 18.67$ & $131.88 \pm 12.42$ & $149.57 \pm 21.35$ & $144.57 \pm 22.95$ & $144.1 \pm 15.08$ \\
TG (mg/dl) & $226.82 \pm 36.94$ & $113.25 \pm 28.38^{\#}$ & $139.28 \pm 29.73^{* *}$ & $131.28 \pm 40.58^{\#}$ & $154.2 \pm 49.62^{* *}$ \\
Ureia (mg/dl) & $38.71 \pm 3.14$ & $42.18 \pm 6.32$ & $37.28 \pm 5.73$ & $40.71 \pm 6.71$ & $40.14 \pm 4.33$ \\
AI & $2.74 \pm 1.11$ & $0.73 \pm 0.30^{\#}$ & $1.39 \pm 0.96^{*}$ & $1.56 \pm 0.88^{*}$ & $0.81 \pm 0.45^{\#}$ \\
\hline
\end{tabular}

Values represent the mean $\pm \mathrm{SD}$ ( $n=7 /$ group), with ${ }^{* *} p<0.01$ and ${ }^{\#} \mathrm{p}<0.001$ representing statistically significant differences compared to the VEI+CSF group. One-Way ANOVA followed by the post-hoc Tukey's test.

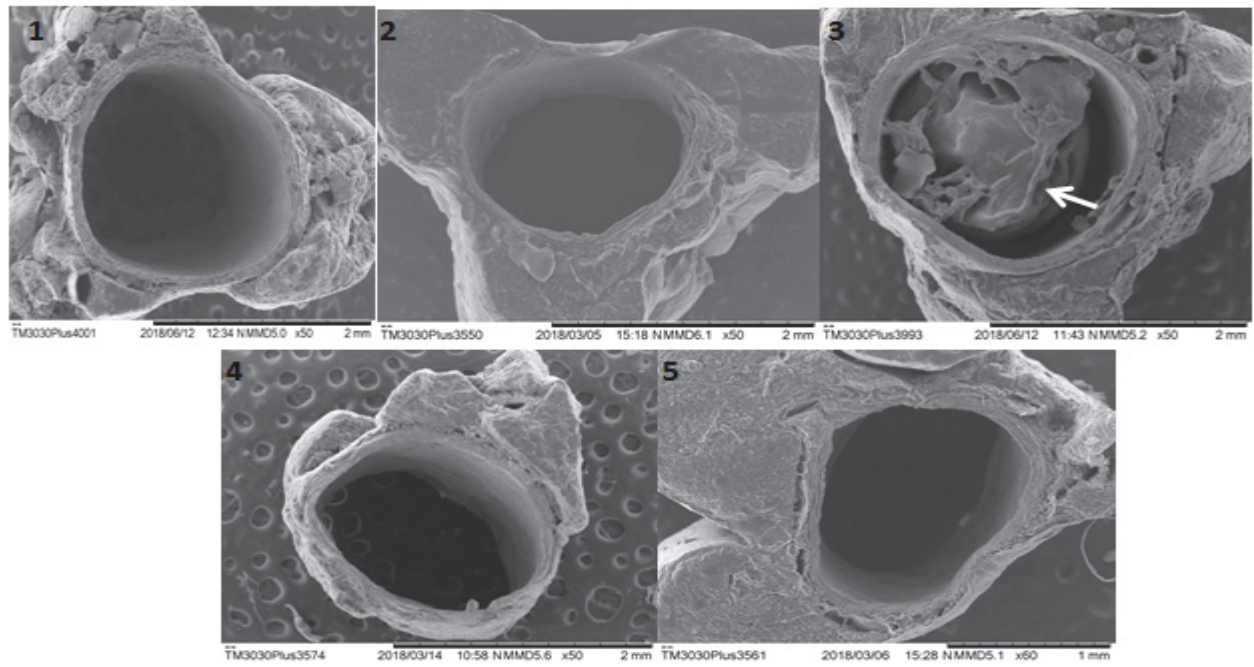

Fig. 6. SEM of transversal sections of the thoracic aorta. (1) EORO; (2) CSF+EORO; (3) CSF+VEI; (4) CSF+NEORO; (5) CSF+SIM. The white arrow indicates the formation of an atheroma plaque in the vascular endothelium.

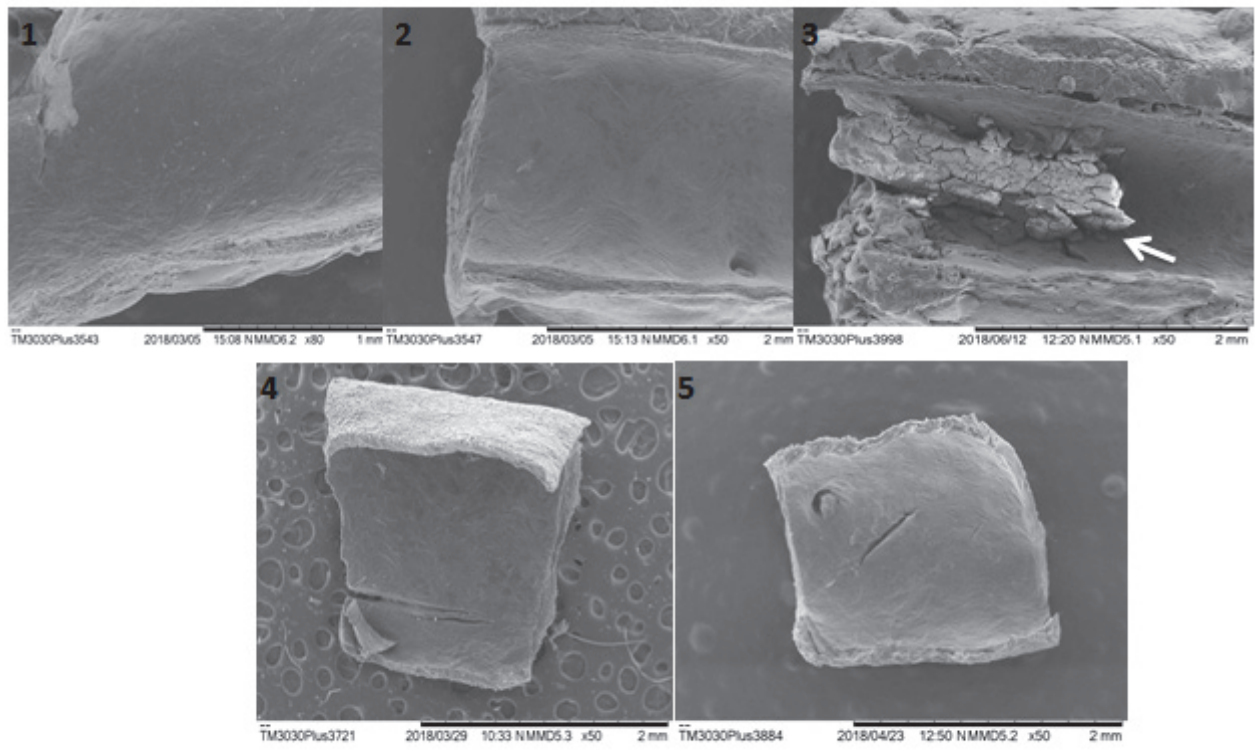

Fig. 7. SEM of longitudinal sections of the thoracic aorta. (1) EORO; (2) CSF+EORO; (3) CSF+VEI; (4) CSF+NEORO; (5) CSF+SIM. The white arrow indicates the formation of an atheroma plaque in the vascular endothelium. 
Then, EORO was used in the development of a nanoemulsion (NEORO). Our results show that NEORO exhibited droplet stability, with average droplets with size $<150 \mathrm{~nm}$ in a monomodal-type distribution (Solé et al., 2012). These results are similar to those reported by Duarte et al. (2015), who developed a stable nanoemulsion from the essential oil of $R$. officinalis, with droplets diameter below $200 \mathrm{~nm}$ and a polydispersity index around 0.280 .

We tested both EORO and NEORO against dyslipidemia. There are several animal models of dyslipidemia, including the classic method induced by intraperitoneal application of Triton (Tyloxapol). Triton is a non-anionic surfactant that induces dyslipidemia by inhibiting lipoprotein lipase, which hydrolyzes triglycerides from serum lipoproteins. Besides, Triton also induces HMA-CoA reductase, an intracellular enzyme that plays a crucial role in the synthesis of hepatic cholesterol. Hence, there is an increase in triglycerides levels (TG) concomitantly with increased total cholesterol (TC) synthesis, resulting in higher serum lipid levels (Souza et al., 2017a; Zarzecki et al., 2014).

In the dyslipidemic groups treated with EORO, NEORO, and simvastatin (positive control), it was observed a decrease of serum lipid levels. This is similar to the report of Lee et al. (2018), who showed serum lipid levels reduction by the essential oil of Citrus lemon. Our results show that treatment either with EORO or NEORO could improve serum lipid profile by decreasing LDL levels and increasing HDL levels in rats. The high content of limonene - one of the major compounds of our oil could partially explain this effect, as this molecule can activate $\operatorname{PPAR} \alpha$ (Jing et al., 2013). PPAR $\alpha$ has a key role in dyslipidemia improvement through the regulation of lipid and lipoprotein metabolism. This was demonstrated through PPAR $\alpha$ activation by an agonist - such as fenofibrate - that can reduce elevated serum lipid levels and increase HDL levels (Yoon, 2009).

Moreover, there are reports on the literature about the anti-atherogenic effect of 1,8-cineole, the major compound of our oil. Jin et al. (2011) reported that the aqueous extract of Turmeric and Laurel - composed of $67 \%$ and $65 \%$ of 1,8 -cineole - decreased TC and TG levels in zebrafish treated with a high cholesterol diet. The extract also protected LDL from oxidation and the cellular uptake of oxidized LDL in vitro and decreased the activity of the enzyme Cholesteryl Ester Transfer Protein (CETP). Cho (2012) further showed that the same could be observed using pure 1,8-cineole. The author reported that 1,8-cineole could improve HDL capacity and inhibit the oxidation of lipoproteins in vitro; in vivo, the author reported that the treatment with 1,8-cineole decreased the levels of serum amyloid, IL-6, and the accumulation of fat in the liver of zebrafish. Finally, one placebo-controlled study in humans reported that capsules of Bay leaves - $58 \%$ of 1,8 -cineole - could decrease the risk for type 2 diabetes and cardiovascular diseases. All three doses tested (1, 2, and $3 \mathrm{~g}$ ) reduced TC levels from $20 \%$ to $24 \%$, reduced LDL levels from $32 \%$ to $40 \%$, reduced TG levels from 25\% and 34\%, and increased HDL levels from $20 \%$ and 29\% (Khan et al., 2009).

A lipid-rich diet contributes to increasing free fatty acid levels in the bloodstream, and increased serum saturated fatty acid is an essential factor in the development of dyslipidemia, atherogenesis, and cardiovascular diseases. The CSF-induced dyslipidemia model entails increased blood lipids levels due to the high content of saturated fat, contributing to hypertriglyceridemia, hypercholesterolemia, increased serum LDL levels, and abdominal fat accumulation (Souza et al., 2017a).

A study performed by Souza et al. (2017b) reported increased abdominal fat in a non-treated group with CSF-in- duced dyslipidemia, just as observed in the group CSF+VEI of our study, corroborating our model. On the other hand, the groups treated with EORO and NEORO had no abdominal fat gain, suggesting a link between these treatments and diminishing abdominal fat. Body fat accumulation is a risk factor for diabetes and cardiovascular diseases due to an imbalance of lipogenic and lipolytic processes that results in excessive storage of fat as triglycerides, mainly in the liver and abdominal adipose tissue (Hassani et al., 2016; Langin et al., 2006).

Evaluating the effects of Rosmarinus officinalis essential oil in diabetic rats, Selmi et al. (2017) reported significantly reduced TC, TG, and LDL levels, without affecting HDL levels. This is in line with our results where the groups CSF+EORO and CSF+NEORO had reduced TC, TG, and LDL levels, without significant alterations of HDL levels. An additional study using essential oil of Ocimun sanctum L., also composed of mono and sesquiterpenes, reported a reduction of TC, TG, and LDL levels (Suanarusawat et al., 2010).

A study performed by Dong et al. (2018) evidenced that the improvement of lipid profile is linked to a reduced incidence of atherosclerosis. The formation of atherogenic plaques is triggered by vascular endothelium injury caused by an increased concentration of serum LDL that enters the arteries' tunica intima and undergoes oxidation, causing inflammation and recruitment of immune system cells, especially monocytes (Souza et al., 2017b). The influx of inflammatory cells to subendothelial space causes their differentiation into macrophages, which absorb the oxidized LDL, become filled with fat, and become foam cells, the main components of atheromas (Blasi, 2008).

Experimental atherosclerosis studies show that a saturated fat-rich diet speeds up the atherogenic process, especially in the abdominal area of the aorta, which is prone to the formation of plaques (Souza et al., 2017b; Jackson et al., 2007). The atherogenesis assessment of the aorta evidences a potential anti-atherogenic effect of EORO and NEORO due to the absence of a plaque formation in the vascular endothelium of treated groups CSF+EORO and CSF+NEORO; this was further corroborated by the atherogenic index values shown in Table 4. That could be explained by EORO's reducing effect over TC, TG, and mainly LDL levels - an essential lipoprotein for atherogenesis.

Borges et al. (2017) demonstrated the anti-inflammatory potential of $R$. officinalis essential oil and its nanoemulsion, which also can contribute by inhibiting the inflammatory cascade that triggers inflammatory cells influx into the subendothelial region. Moreover, the antioxidant activity of EORO may also contribute inhibiting atherogenesis by suppressing LDL oxidation, preventing its absorption by macrophages, and hence preventing the formation of foam cells (Rašković et al., 2014).

\section{Conclusions}

Based on these results, we report that EORO $(100 \mathrm{mg} / \mathrm{kg})$ and NEORO $(500 \mu \mathrm{g} / \mathrm{kg})$ could significantly reduce the levels of total cholesterol, triglycerides, and LDL in Triton and CSF-induced dyslipidemia models, among other benefits. NEORO efficiently improved the lipid profile with a dose 200 times smaller than the solution of EORO. The anti-atherogenic capacity of EORO and NEORO was also shown by the absence of atheroma plaque formation in the aorta, assessed by scanning electron microscopy and the atherogenic index. Overall, in the Triton-induced dyslipidemia model, EORO treatment had su- 
perior values than NEORO's (and simvastatin), although the differences were not too high, while in the CSF model, the values were mixed. The anti-atherogenic potential of EORO can be explained at least partially by pharmacological mechanisms already described from its major compounds, such as LDL level reducer, antioxidant and anti-inflammatory.

\section{Authors' contributions}

APSR, BSFS, ASAB, and HOC performed all the experiments of the dyslipidemia study. JL and CPF performed the obtaining and analysis of NEORO. LMB and RB performed the Biochemical analysis. AMF, IMF, and ACMP performed the Phytochemistry study and JCTC was involved in drafting, correcting the manuscript, and coordinated the study. All the authors read the manuscript, critically revised it for relevant intellectual content, and approved the final version of the manuscript.

\section{Conflict of interests}

The authors declare that they have no conflict of interests.

\section{Acknowledgements}

The authors would like to thank CAPES (no 3292/2013 AUXPE), CNPq Proc. 402332/2013-0 and FAPEAP (No 003/2018 Programa de Pesquisa para o SUS/FAPEAP/MS-DECIT/CNPq/ SESA-AP) for the financial support and Fundação de Amparo a Pesquisa do Estado do Amapá - FAPEAP for the APSR scholarship.

\section{References}

Affholder M-C, Prudent P, Masotti V, Coulomb B, Rabier J, Nguyen B, et al. (2013). Transfer of metals and metalloids from soil to shoots in wild rosemary (Rosmarinus officinalis L.) growing on a former lead smelter site: Human exposure risk. Sci Total Environ 454: 219-229. DOI: 10.1016/j.scitotenv.2013.02.086.

Blasi C (2008). The autoimmune origin of atherosclerosis. Atherosclerosis 201: 17-32. DOI: 10.1016/j. atherosclerosis.2008.05.025.

Bonfim MR, Oliveira ASB, Amaral SL, Monteiro HL (2015). Tratamento das Dislipidemias com Estatinas e Exercícios Físicos: Evidências Recentes das Respostas Musculares. Arq Bras Cardiol 104: 324-332. DOI: 10.5935/abc.20150005.

Borges RS, Lima ES, Keita H, Ferreira IM, Fernandes CP, Cruz RAS, et al. (2017). Anti-inflammatory and antialgic actions of a nanoemulsion of Rosmarinus officinalis L. essential oil and a molecular docking study of its major chemical constituents. Inflammopharmacology 26: 1-13. DOI: 10.1007/s10787-0170374-8.

Boullart ACI, De Graaf J, Stalenhoef AF (2012). Triglicerídeos séricos e risco de doença cardiovascular. Biochimica et Biophysica Acta (BBA) - Molecular and Cell Biology of Lipids 1821(5): 867-875. DOI: 10.1016/j.bbalip.2011.10.002.

Brea Hernando ÁJ (2014). Dianas terapéuticas en el tratamiento de las dislipemias: colesterol no unido a lipoproteínas de alta densidad y colesterol unido a lipoproteínas de alta densidad. Clínica e investigación en arteriosclerosis. 26: 3-6. DOI: 10.1016/ S0214-9168(14)70018-X.

Carvalho HO, Souza BSF, Santos IVF, Resque RL, Keita H, Fernandes CP, et al. (2016). Hypoglycemic effect of formulation containing hydroethanolic extract of Calophyllum brasiliense in diabetic rats induced by streptozotocin. Rev Bras Farmacogn 26: 10-18. DOI: 10.1016/j.bjp.2016.04.004.

Cho KH (2012). 1, 8-cineole protected human lipoproteins from modification by oxidation and glycation and exhibited serum lipidlowering and anti-inflammatory activity in zebrafish. BMB Rep 45(10): 565-570. DOI: 10.5483/BMBRep.2012.45.10.044.
Cleff MB, Meinerz ARM, Madrid I, Fonseca AO, Alves GH, et al. (2012). Perfil de suscetibilidade de leveduras do gênero Candida isoladas de animais ao óleo essencial de Rosmarinus officinalis L. Rev Bras Plantas Med 14: 43-49. DOI: 10.1590/S151605722012000100007.

Dobiášová M (2004). Atherogenic index of plasma [log (triglycerides/ HD-cholesterol)]: theoretical and practical implications. Clin Chem 50(7): 1113-1115. DOI: 10.1373/clinchem.2004.033175.

Dong Z, Shi H, Zhao M, Zhang X, Huang W, Wang Y, et al. (2018). Loss of LCAT activity in the golden Syrian hamster elicits pro-atherogenic dyslipidemia and enhanced atherosclerosis. Metabolism 83: 245-255. DOI: 10.1016/j.metabol.2018.03.003.

Dowla S, Aslibekyan S, Goss A, Fontaine K, Ashraf AP (2018). Dyslipidemia is associated with pediatric nonalcoholic fatty liver disease. J Clin Lipidol 12(4): 981-987. DOI: 10.1016/j. jacl.2018.03.089.

Duarte JL, Amado JRR, Oliveira AEMFM, Cruz RAS, Ferreira AM, Souto RNP, et al. (2015). Evaluation of larvicidal activity of a nanoemulsion of Rosmarinus officinalis essential oil. Rev Bras Farmacogn 25: 189-189. DOI: 10.1016/j.bjp.2015.02.010.

Fernandes CP, Mascarenhas MP, Zibetti FM, Lima BG, Oliveira RPRF, Rocha L (2013). HLB value, an importante parameter for the development of essential oil phytopharmaceuticals. Braz J Pharm 23: 108-114. DOI: 10.1590/S0102-695X2012005000127.

Handayani R, Sulistyo J, Rahayu RD (2009). Extraction of coconut oil (Cocos nucifera L.) through fermentation system. Biodiversitas 10: 3. DOI: 10.13057/biodiv/d100309.

Hassani FV, Shirani K, Hosseinzadeh H (2016). Rosemary (Rosmarinus officinalis) as a potential therapeutic plant in metabolic syndrome: a review. Naunyn Schmiedeberg's Arch Pharmacol 389: 931-949. DOI: 10.1007/S00210-016-1256-0.

Jackson CL, Bennett MR, Biessen EAL, Johnson J.L, Krams RR (2007). Assessment of unstable atherosclerosis in mice. Am Heart Assoc 27: 714-720. DOI. 10.1161/01.ATV.0000261873.86623.e1.

Jin S, Hong JH, Jung SH, Cho KH (2011). Turmeric and laurel aqueous extracts exhibit in vitro anti-atherosclerotic activity and in vivo hypolipidemic effects in a zebrafish model. J Med Food 14(3): 247-256. DOI: 10.1089/jmf.2009.1389.

Jing L, Zhang Y, Fan S, Gu M, Guan Y, Lu X, et al. (2013). Preventive and ameliorating effects of citrus D-limonene on dyslipidemia andhyperglycemia in mice with high-fat diet-induced obesity. Eur J Pharmacol 715: 46-55. DOI: 10.1016/j.ejphar.2013.06.022.

Khan A, Zaman G, Anderson RA (2009). Bay leaves improve glucose and lipid profile of people with type 2 diabetes. J Clin Bioch Nutr 44(1): 52-56. DOI: $10.3164 /$ jcbn.08-188.

Langin D (2006). Adipose tissue lipolysis as a metabolic pathway to define pharmacological strategies against obesity and the metabolic syndrome. Pharmacol Res 53: 482-491. DOI: 10.1016/j. phrs.2006.03.009.

Lee H, Woo M, Kim M, Noh JS, Song YO (2018). Antioxidative and Cholesterol-Lowering Effects of Lemon Essential Oil in Hypercholesterolemia-Induced Rabbits. Prev Nutr Food Sci 23: 8. DOI: 10.3746/pnf.2018.23.1.8.

Lin CF, Chang YH, Chien SC, YH (2018). Epidemiology of Dyslipidemia in the Asia Pacific Region. Int J Gerontol 12: 2-6. DOI: 10.1016/j.ijge.2018.02.010.

Ostertag F, Weiss J, McClements DJ (2012). Low-energy formation of edible nanoemulsions: factors influencing droplet size produced by emulsion phase inversion. J Colloid Interface Sci 388: 95-102. DOI: 10.1016/j.jcis.2012.07.089.

Rašković A, Milanović I, Pavlović N, Ćebović T, Vukmirovic S, Mikov M (2014). Antioxidant activity of rosemary (Rosmarinus officinalis L.) essential oil and its hepatoprotective potential. BMC Complement Alter Med 14: 225. DOI: 10.1186/1472-6882-14225.

Satyal P, Jonest H, Lopez EM, McFeeters RL, Ali NAA, Mansi I, et al. (2017). Chemotypic Characterization and Biological Activity of Rosmarinus officinalis. Foods 6: 20. DOI: 10.3390/foods6030020.

Sedighi R, Zhao YA, Sang S (2015). Preventive and protective properties of rosemary (Rosmarinus officinalis L.) in obesity and diabetes mellitus of metabolic disorders: a brief review. Current Opinion in Food Science 2: 58-70. DOI: 10.1016/j. cofs.2015.02.002. 
Selmi S, Rtibi K, Grami D, Sebai H, Marzouki L (2017). Rosemary (Rosmarinus officinalis) essential oil components exhibit antihyperglycemic, anti-hyperlipidemic and antioxidant effects in experimental diabetes. Pathophysiology 24: 297-303. DOI: $10.1016 /$ j.pathophys.2017.08.002.

Solé I, Solans C, Maestro A, González C, Gutiérrez JM (2012). Study of nanoemulsion formation by dilution of microemulsions. J Colloid Interface Sci 376: 133-139. DOI: 10.1016/j. jcis.2012.02.063.

Souza BSF, Carvalho HO, Ferreira IM, da Cunha EL, Barros AS, Taglialegna T, Carvalho JCT (2017a). Effect of the treatment with Euterpe oleracea Mart. oil in rats with Triton-induced dyslipidemia. Biomed Pharmacother 90: 542-547. DOI: 10.1016/j. biopha.2017.04.005.

Souza BSF, Carvalho HO, Taglialegna T, Barros ASA, da Cunha EL, Ferreira IM, et al. (2017b). Effect of Euterpe oleracea Mart. (Açaí) Oil on Dyslipidemia Caused by Cocos nucifera L. Saturated Fat in Wistar Rats. J Med Food 20: 830-837. DOI: $10.1089 / \mathrm{jmf} .2017 .0027$.
Suanarunsawat T, Ayutthaya WDN, Songsak T, Thirawarapan S, Poungshompoo S (2010). Antioxidant Activity and Lipid-Lowering Effect of Essential Oils Extracted from Ocimum sanctum L. Leaves in Rats Fed with a High Cholesterol Die. J Clin Biochem Nutr 46: 52-59. DOI: 10.3164/jcbn.09-52.

Takayama C, Faria FM, Almeida ACA, Dunder RJ, Manzo LP, Socca EAR, et al. (2016). Chemical composition of Rosmarinus officinalis essential oil and antioxidant action against gastric damage induced by absolute ethanol in the rat. Asian Pacific Journal of Tropical Biomedicine 6: 677-681. DOI: 10.1016/j. apjtb.2015.09.027.

Yoon M (2009). The role of PPAR $\alpha$ in lipid metabolism and obesity: focusing on the effects of estrogen on PPAR $\alpha$ actions. Pharmacol Res 60: 151-159. DOI: 10.1016/j.phrs.2009.02.004.

Zarzecki MS, Araujo SM, Bortolotto VC, de Paula MT, Jesse CR, Prigol M (2014). Hypolipidemic action of chrysin on Triton WR1339-induced hyperlipidemia in female C57BL/6 mice. Toxicol Rep 1: 200-208. DOI: 10.1016/j.toxrep.2014.02.003. 